\title{
ILM peeling with ILM flap transposition vs. classic ILM peeling for small and medium macula holes-a prospective randomized trial
}

\author{
Christoph Leisser · Manuel Ruiss · Caroline Pilwachs · Oliver Findl
}

Received: 1 December 2021 / Accepted: 12 January 2022 / Published online: 23 February 2022

(c) The Author(s), under exclusive licence to Springer-Verlag GmbH Austria, ein Teil von Springer Nature 2022

\begin{abstract}
Summary
Background The aim of the present study was to compare macular hole closure rates of patients with small and medium-sized macular holes who underwent vitrectomy with internal limiting membrane (ILM) peeling combined with ILM flap transposition over the macular hole, compared with classic ILM peeling. Methods This prospective randomized trial was designed as a pilot study with two groups: ILM peeling with ILM flap transposition over the macular hole vs. classic ILM peeling.

Results Among 20 patients recruited, complete analysis could be performed for 16 patients. The macular hole closure rates were $100 \%$ in both groups, without significant differences with respect to postsurgical subfoveal hyporeflective zones ( $p=1.0$, Fisher's exact test), postsurgical visual acuity ( $p=0.7, t$-test), and postsurgical irregularities of the ellipsoid zone ( $p=1.0$, Fisher's exact test).

Conclusion Vitrectomy with ILM peeling combined with ILM flap transposition over the macular hole and classic ILM peeling are both successful methods for the repair of macular holes of small and medium size and are associated with comparable outcomes.
\end{abstract}

Keywords Idiopathic full thickness macular holes · Vitrectomy with ILM peeling - ILM flap transposition · Gastamponade - Small and medium size macular holes
ILM-Peeling mit ILM-Flap-Transposition vs. klassisches ILM-Peeling für kleine und mittlere Makulaforamina - eine prospektive randomisierte Studie

\section{Zusammenfassung}

Hintergrund Das Ziel der Studie war ein Vergleich der Verschlussraten von Makulaforamina bei Patienten mit kleinen und mittleren Makulaforamina bei Vitrektomie mit Peeling der Membrana limitans interna (,internal limiting membrane“ [ILM]), kombiniert mit einer Transposition eines ILM-Flaps über das Makulaforamen, verglichen mit dem klassischen ILM-Peeling. Methoden Die vorliegende prospektive randomisierte Studie wurde als Pilotstudie mit 2 Gruppen (ILMPeeling mit ILM-Flap-Transposition über das Makulaforamen vs. klassisches ILM-Peeling) geplant.

Ergebnisse Von 20 rekrutierten Patienten konnte die Analyse der Resultate bei 16 Patienten durchgeführt werden. Es zeigte sich eine Verschlussrate der Makulaforamina von $100 \%$ in beiden Gruppen, ohne signifikante Unterschiede in Bezug auf postoperative hyporeflektive Zonen ( $p=1,0$; Fisher-Exact-Test), postoperativen Visus ( $p=0,7 ; t$-Test) und postoperative Irregularitäten in der ellipsoiden Zone ( $p=1,0$; Fisher-ExactTest).

Schlussfolgerung Die Vitrektomie mit ILM-Peeling, kombiniert mit ILM-Flap-Transposition über das Makulaforamen, und das klassische ILM-Peeling sind beides erfolgreiche Methoden bei kleinen und mittleren Makulaforamina mit vergleichbaren Ergebnissen.

Schlüsselwörter Idiopathisches Makulaforamen .

Vitrektomie mit ILM-Peeling · ILM-Flap-

Transposition · Gastamponade $\cdot$ Kleine und mittlere Makulaforamina 


\section{Introduction}

Idiopathic macular holes, hypothesized to be induced by anteroposterior traction of the vitreous on the fovea [1] and/or vitreoschisis [2], are full-thickness defects of the fovea leading to central scotomata and deterioration of vision. Kelly and Wendel were the first to describe vitrectomy as a promising treatment option for surgical repair of MHs [3], and additional internal limiting membrane (ILM) peeling with gas or air tamponade has improved outcomes further [4-6]. Nevertheless, MH closure rates of large MHs was still poor until the introduction of the inverted ILM flap techniques $[7,8]$.

To date, there is a controversy with respect to the best technique of ILM flap surgery. Michalewska et al. described the temporal inverted ILM flap technique, without peeling of the superior, nasal, and inferior ILM around the $\mathrm{MH}$ [8], in contrast to the surgical technique described by Shin et al., who observed a case of failure of $\mathrm{MH}$ closure due to residual ILM at the $\mathrm{MH}$ margin and adapted their technique to a combined ILM peeling and ILM flap technique [9].

In the $\mathrm{MH}$ classification scheme of the International Vitreomacular Traction Study Group [10], small MHs are defined with a minimal $\mathrm{MH}$ diameter of $250 \mu \mathrm{m}$ or smaller, medium MHs with a minimal $\mathrm{MH}$ diameter up to $400 \mu \mathrm{m}$, and large $\mathrm{MHs}$ with a minimal MH diameter larger than $400 \mu \mathrm{m}$.

Combination of ILM peeling and ILM flap transposition over the $\mathrm{MH}$ offers relief of tangential traction on the retinal surface with ILM peeling and a scaffold for migrating Müller cells along the ILM flap to close the MH. Furthermore, ILM peeling is hypothesized to induce activation of Müller cells, and ILM flaps are rich in neurotrophic factors to stimulate migration of Müller cells for $\mathrm{MH}$ closure and retina regeneration $[11,12]$. The combination of ILM peeling and ILM flap transposition over the $\mathrm{MH}$ can be easily performed without use of perfluoro- $n$-octane (use of heavy liquids was described by Shin et al. [9]), since air or gas tamponade holds the ILM flap in the correct position over the $\mathrm{MH}$ after fluid/air exchange, resulting in high $\mathrm{MH}$ closure rates [13, 14].

The aim of the present study was to compare $\mathrm{MH}$ closure rates in patients with small and medium MHs, who underwent vitrectomy with ILM peeling combined with ILM flap transposition over the MH, compared with classic ILM peeling.

\section{Patients and methods}

This prospective study included patients scheduled for surgical repair of a small or medium MH between September 2018 and January 2021 at the Department of Ophthalmology at the Hanusch Hospital in Vienna, Austria. All research and measurements followed the tenets of The Declaration of Helsinki and were approved by the local ethics committee of the city of Vi-

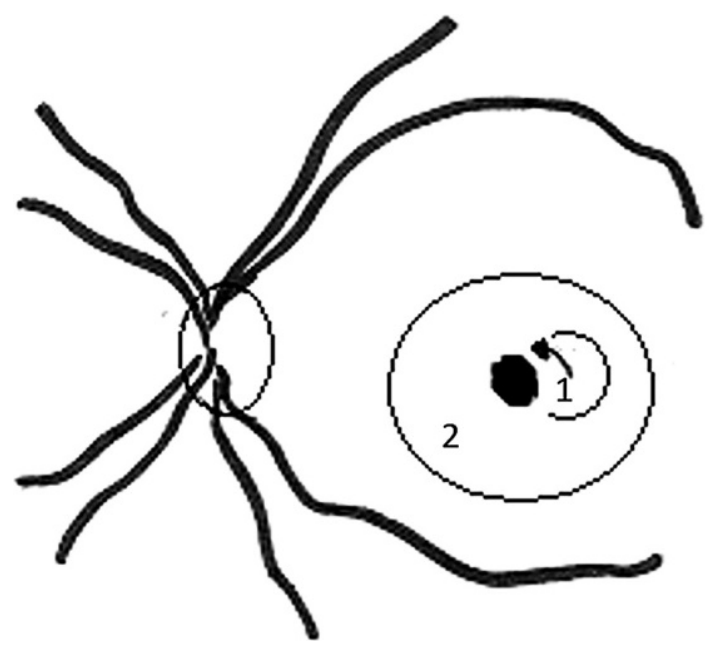

Fig. 1 Surgical technique of internal limiting membrane (ILM) peeling with ILM flap transposition over the macular hole (MH). A temporal ILM flap was prepared (1), while the residual ILM around the $\mathrm{MH}$ was peeled until the rim of the $\mathrm{MH}(2)$, after which the ILM flap was positioned in an inverted fashion over the $\mathrm{MH}$ (arrow)

enna (EK 18-160-0818; Clinical trials registration: NCT 03646695).

The inclusion criteria were: (a) presence of a fullthickness idiopathic $\mathrm{MH}$ with a minimal $\mathrm{MH}$ diameter smaller than $400 \mu \mathrm{m}$, (b) age of 18 years or older, and (c) informed consent for study participation. Patients with impending MHs were excluded from study participation; furthermore, there were no patients with high myopia included in the study.

Patients were allocated to the following groups by randomization with sealed envelopes: (a) ILM peeling with ILM flap transposition over the MH (ILM flap group), or (b) classic ILM peeling (ILM peeling group). The study was designed as a pilot study with 20 participants, and calculation of sample size was planned with the results of this pilot study.

Surgery was performed with $23-\mathrm{G}$ pars plana vitrectomy according to presurgical randomization with (a) combined ILM peeling with ILM flap transposition over the MH (ILM flap group, Fig. 1), or (b) classic ILM peeling (ILM peeling group). All surgeries were performed by the same vitreo-retinal surgeon. For ILM visualization, chromovitrectomy with a trypan blue and brilliant blue G-based dye (MembraneBlue-Dual, D.O.R.C., The Netherlands) was performed and the ILM was peeled using an end-gripping forceps. After successful peeling, fluid-air exchange was performed and for those in the ILM flap group, correct ILM flap positioning over the $\mathrm{MH}$ was checked. All patients received intraocular gas tamponade with sulfur hexafluoride $20 \%$ (Alchimia S.R.L, Italy) and were encouraged to remain in postoperative face-down positioning for at least $48 \mathrm{~h}$ after surgery.

Optical coherence tomography (OCT) imaging of the macula was performed with a stand-alone spectral domain-OCT (SD-OCT) device (Cirrus HD-OCT, 
Carl Zeiss Meditec AG, Germany) before surgery, and 3 months after surgery. The minimal diameter of the $\mathrm{MH}$ was measured on the horizonal foveal SD-OCT scans. Measurements were performed with the caliper function of the SD-OCT device. Distance-corrected visual acuity (DCVA) was determined at the same time points using EDTRS charts (Precision Vision, USA) at a distance of $4 \mathrm{~m}$ by masked examiners. All patients received nonsteroidal and steroidal anti-inflammatory eye drops during the first month after surgery.

Combined phacoemulsification with implantation of an intraocular lens and 23-G pars plana vitrectomy was performed in cases of coexisting vision-affecting cataract.

Successful closure of MH was defined as a type 1 closure, according to Kang et al. [15], with restoration of foveal tissue, while type 2 closure, without restoration of foveal tissue, is typically associated with poor improvement in visual acuity and was regarded as failure.

\section{Statistical analysis}

Statistical analysis was performed in a descriptive fashion for mean value, standard deviation, median, interquartile range (IQR) and range. All data were tested for normal distribution using the Kolmogorov-Smirnoff test. In the case of a normal distribution, mean and standard deviation were calculated and otherwise median, IQR, and range were determined. The $t$-test was used for normally distributed data, otherwise the Mann-Whitney $U$-test was used, and for dichotomic data, Fisher's exact test was used. Regression analysis was performed as a multiple regression analysis with elimination of factors with values of $p>0.05$, and sample size calculation was performed with the chi-square test. A value of $p<0.05$ was regarded to indicate significant differences between groups. Statistical analysis was performed using the software tool BiAS (epsilon Verlag, Germany).

\section{Results}

In total, 20 patients were recruited for the study. Four patients had to be excluded from analysis, as two of them were lost to follow-up (one patient died from cancer in the follow-up period, and the other patient did not want to come to the follow-up examination 3 months after surgery), one patient canceled the surgery, and one patient had a loss of the ILM flap during fluid/air exchange (nevertheless, with successful MH closure). Due to COVID 19-related restrictions, two patients had their follow-up examination later than at 3 months after surgery (at 6 months and at 7 months after surgery, respectively). As the main outcome was defined to be the $\mathrm{MH}$ closure rate, a criterion not being influenced by the time point of fol-
Table 1 Demographic data and postsurgical results

\begin{tabular}{|c|c|c|}
\hline $\begin{array}{l}\text { Demographic } \\
\text { data and surgical } \\
\text { results }\end{array}$ & Classic ILM peeling & $\begin{array}{l}\text { ILM peeling with ILM flap } \\
\text { transposition }\end{array}$ \\
\hline $\begin{array}{l}\text { Number of } \\
\text { patients }\end{array}$ & 9 & 7 \\
\hline $\begin{array}{l}\text { Mean age of } \\
\text { patients }\end{array}$ & 67 years (SD: \pm 5 ) & 71 years (SD: \pm 7 ) \\
\hline Gender & Females: 7/males: 2 & Females: 4/males: 3 \\
\hline Eye & Right: 5/left: 4 & Right: 3/left: 4 \\
\hline $\begin{array}{l}\text { Mean minimal MH } \\
\text { diameter }\end{array}$ & $244 \mu \mathrm{m}$ (SD: \pm 101$)$ & $275 \mu \mathrm{m}(\mathrm{SD}: \pm 90)$ \\
\hline $\begin{array}{l}\text { Mean basal MH } \\
\text { diameter }\end{array}$ & $679 \mu \mathrm{m}(\mathrm{SD}: \pm 273)$ & $634 \mu \mathrm{m}(\mathrm{SD}: \pm 208)$ \\
\hline \multicolumn{3}{|l|}{ Lens status } \\
\hline Phakic & 8 & 4 \\
\hline Pseudophakic & 0 & 1 \\
\hline Phacovitrectomy & 1 & 1 \\
\hline Coexisting VMA & 5 & 2 \\
\hline \multicolumn{3}{|l|}{ Visual acuity } \\
\hline $\begin{array}{l}\text { Median presur- } \\
\text { gical DCVA }\end{array}$ & $\begin{array}{l}20 \text { EDTRS letters } \\
\text { (IQR: 6-24, range: } 3-33 \text { ) }\end{array}$ & $\begin{array}{l}18 \text { EDTRS letters } \\
\text { (IQR: } 17-23 \text {, range: } 15-30)\end{array}$ \\
\hline $\begin{array}{l}\text { Mean postsurgical } \\
\text { DCVA } \\
\text { (median postsurgi- } \\
\text { cal DCVA) }\end{array}$ & $\begin{array}{l}38 \text { EDTRS letters (SD: } \pm 5 \text { ) } \\
\text { (38 EDTRS letters, IQR: } \\
\text { 34-44, range: } 30-45 \text { ) }\end{array}$ & $\begin{array}{l}40 \text { EDTRS letters (SD: } \pm 7 \text { ) } \\
\text { (38 EDTRS letters, IQR: } \\
\text { 34-44, range: } 30-50 \text { ) }\end{array}$ \\
\hline MH closure & All patients & All patients \\
\hline
\end{tabular}

low-up, we decided to include these patients in the analysis. Demographic data are provided in Table 1.

All patients $(n=16)$ had successful MH closure after surgery (Fig. 2), without differences between groups, and there were no significant differences between the groups with respect to occurrence of postsurgical subfoveal hyporeflective zones (ILM peeling group: $22 \%$ with subfoveal hyporeflective zones, ILM flap group: $14 \%$ with subfoveal hyporeflective zones, $p=1.0$; Fisher's exact test). Due to the fact that $\mathrm{MH}$ closure rates were $100 \%$ in both groups after the first surgery, sample size calculation with the chi-square test was not possible after the pilot study.

Improvement of postsurgical visual acuity at the postsurgical follow-up was observed among all patients, with a mean improvement of +21 EDTRS letters (SD: \pm 9 ) in the ILM peeling group, and a mean improvement of +20 EDTRS letters (SD: \pm 7 ) in the ILM flap group. Mean postsurgical DCVA at the final visit was 38 EDTRS letters (SD: \pm 5 ) in the ILM peeling group, and 40 EDTRS letters (SD: \pm 7 ) in the ILM flap group. Differences between groups were not significant (improvement of DCVA: $p=0.7, t$-test; postsurgical DCVA: $p=0.7, t$-test). Due to the fact that $89 \%$ of patients in the ILM peeling group were phakic (among them three patients had vision-affecting cataract at the follow-up), and $57 \%$ of patients in the ILM flap group were phakic (among them two patients 

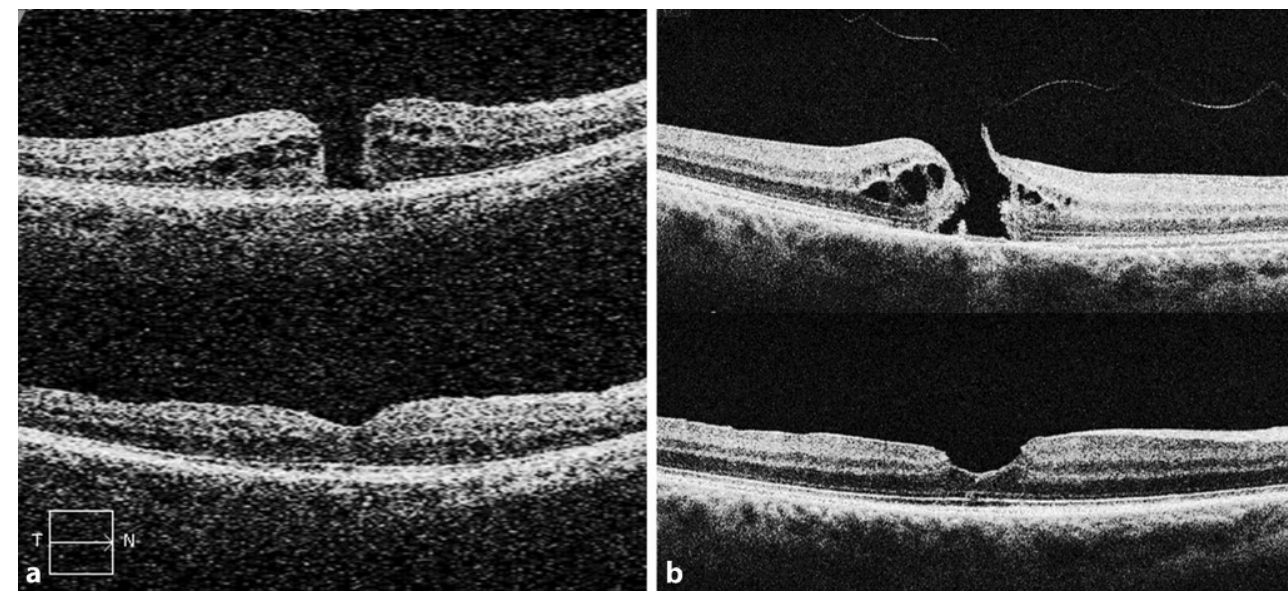

Fig. 2 Presurgical (a) and postsurgical (b) OCT images from internal limiting membrane (ILM) peeling with ILM flap transposition over the macular hole (a: upper panel presurgical, and lower panel postsurgical images 3 months after surgery-distance-corrected visual acuity [DCVA] improved

had vision-affecting cataract at the follow-up), we did not perform sample size calculation with visual acuity data, because of a possible bias of the lens status on the visual acuity outcomes.

Postsurgical irregularities of the ellipsoid zone at the follow-up examination were detected in $56 \%$ $(n=5)$ of patients in the ILM peeling group, and $57 \%$ $(n=4)$ of patients in the ILM flap group (differences between groups were not significant, $p=1.0$, Fisher's exact test). In a multiple regression analysis (with elimination of factors with $p>0.05$ and the predictors: postsurgical irregularities of the ellipsoid zone, phakic lens status, ILM flap transposition, minimal $\mathrm{MH}$ diameter, basal $\mathrm{MH}$ diameter, presurgical DCVA, coexisting presurgical vitreomacular adhesion), postsurgical irregularities of the ellipsoid zone were the only significant factor influencing postsurgical DCVA at the follow-up in our group of patients with small and medium-sized MHs.

The ILM flap in the ILM peeling with ILM flap transposition group was visible in only one patient in the follow-up OCT, without any negative effects on postsurgical DCVA (presurgical DCVA: 17 EDTRS letters, postsurgical DCVA: 44 EDTRS letters).

\section{Discussion}

Vitrectomy with ILM peeling combined with ILM flap transposition over the MH and classic ILM peeling resulted in comparable $\mathrm{MH}$ closure rates. There were no significant differences in postsurgical DCVA, occurrence of subfoveal hyporeflective zones, and irregularities of the ellipsoid zone between groups.

This study was based on the commonly used "cutoff" MH diameter of $400 \mu \mathrm{m}$, as proposed by the International Vitreomacular Traction Study Group [10]. Recently published outcomes of the BEAVRS database (mainly based on classic ILM peeling outcomes) were from 30 EDTRS letters to 50 EDTRS letters after surgery) and classic ILM peeling (b: upper panel presurgical, and lower panel postsurgical images 3 months after surgery-DCVA improved from 17 EDTRS letters to 37 EDTRS letters after surgery)

not available when designing the study. The analysis of the BEAVRS database outlined a cut-off MH diameter of $500 \mu \mathrm{m}$ being better for prediction of postsurgical prognosis [16]. For future studies this new cutoff MH diameter of $500 \mu \mathrm{m}$ should be considered.

It is a common procedure to perform classic ILM peeling in cases of small or medium MHs, and ILM flap techniques in cases of large MHs. Nevertheless, ILM flap techniques are also a possible option for small and medium MHs. Therefore, the main goal of this prospective randomized study was to compare an ILM flap technique (we used ILM peeling with ILM flap transposition over the MH) with classic ILM peeling among small and medium MHs. Despite the high $\mathrm{MH}$ closure rates in this population of patients with both techniques, there was a small risk for anatomical failure in both groups. In the case of using an ILM flap technique, repositioning of the ILM flap in a second surgery would offer high chances for $\mathrm{MH}$ closure, whereas in cases of classic ILM peeling, alternative ILM flap techniques, such as pedicle ILM flap transposition over the MH (first described by Gekka et al. [17]) or free ILM flap insertion into the MH (first described by Morizane et al. [18]), would be an option for a second surgery; however, for both techniques, ILM flaps can be hypothesized to be less stable in position, compared with repositioned temporal ILM flaps.

During vitrectomy with ILM peeling and ILM flap transposition, we usually only positioned ILM flaps over the MH without any tucking of the ILM flap inside the $\mathrm{MH}$, since reports showed that tucking the ILM flap inside the MH did not result in any benefit $[19,20]$. Furthermore, we did not use any heavy liquids (such as perfluoro- $n$-octane) for ILM flap fixation, as proposed by Shin et al. [9], since ILM flaps can be successfully positioned over the MH directly before finishing fluid/air exchange, and with intraoc- 
ular tamponades (air or gas tamponade) the ILM flaps typically remain stable in position over the $\mathrm{MH}$.

Combining ILM peeling with ILM flap transposition follows the experience with ILM flaps reported by Shin et al. [9], who observed risk of failure when leaving ILM at the MH margins. In general, "lack of ILM peel" is regarded to be associated with lower odds of success [21].

To our knowledge, there are two recent publications comparing ILM peeling and ILM flap techniques for small and medium MHs retrospectively $[22,23]$. While Baumann et al. reported no significant differences between groups [22], Chou et al. found better results for the ILM flap group in the earlier postsurgical period (up to 6 months after surgery), but not afterwards [23].

We planned to perform a sample size calculation using the $\mathrm{MH}$ closure rates in this study. Due to the fact that both groups ended up having a $100 \% \mathrm{MH}$ closure rate, sample size calculation with the chi-square test was not possible. An alternative would have been to perform sample size calculations with the vision results, but there was a high number of phakic patients with possible bias in the results due to postsurgical cataract formation. The high number of phakic patients can be explained by the fact that we only perform phacovitrectomy in cases of coexisting presurgical vision-affecting cataract.

\section{Conclusion}

Vitrectomy with ILM peeling combined with ILM flap transposition over the $\mathrm{MH}$ and classic ILM peeling are both successful methods for repair of small and medium $\mathrm{MH}$ with comparable outcomes.

Conflict of interest $\mathrm{O}$. Findl is a scientific advisor for Alcon, Croma, Carl Zeiss Meditec AG, Johnson \& Johnson and Merck, but has no personal interest in the products mentioned. C. Leisser, M. Ruiss and C. Pilwachs declare that they have no competing interests.

\section{References}

1. Steel DH, Lotery AJ. Idiopathic vitreomacular traction and macular hole: a comprehensive review of pathophysiology, diagnosis, and treatment. Eye (Lond). 2013;27:S1-21. https://doi.org/10.1038/eye.2013.212.

2. Sebag J, Gupta P, Rosen RR, et al. Macular holes and macular pucker: the role of vitreoschisis as imaged by optical coherence tomography/scanning laser ophthalmoscopy. Trans Am Ophthalmol Soc. 2007;105:121-9.

3. Kelly NE, Wendel RT. Vitreous surgery for idiopathic macular holes. Results of a pilot study. Arch Ophthalmol. 1991;109:654-9. https://doi.org/10.1001/archopht.1991. 01080050068031.

4. Eckardt C, Eckardt U, Groos S, et al. Entfernung der Membrana limitans interna bei Makulalöchern. Klinische und morphologische Befunde. Ophthalmologe. 1997;94:545-51. https://doi.org/10.1007/s003470050156.

5. ParkDW, Sipperley JO, Sneed SR, et al. Macular hole surgery with internal-limiting membrane peeling and intravitreous air. Ophthalmology. 1999;106:1392-7. https://doi.org/10. 1016/S0161-6420(99)00730-7.

6. Brooks HL Jr.. Macular hole surgery with and without internal limiting membrane peeling. Ophthalmology. 2000;107:1939-48. https://doi.org/10.1016/s01616420(00)00331-6.

7. Michalewska Z, Michalewski J, Adelman RA, et al. Inverted internal limiting membrane flap technique for large macular holes. Ophthalmology. 2010;117:2018-25. https://doi. org/10.1016/j.ophtha.2010.02.011.

8. Michalewska Z, Michalewski J, Dulczewska-Cichecka K, et al. Temporal inverted internal limiting membrane flap technique versus classic inverted internal limiting membrane flap technique: a comparative study. Retina. 2015;35:1844-50. https://doi.org/10.1097/IAE. 0000000000000555.

9. Shin MK, Park KH, Park SW, et al. Perfluoro-n-octane-assisted single-layered inverted internal limiting membrane flap technique for macular hole surgery. Retina. 2014;34:1905-10. https://doi.org/10.1097/IAE. 0000000000000339.

10. Duker JS, Kaiser PK, Binder S, et al. The international vitreomacular traction study group classification of vitreomacular adhesion, traction, and macular hole. Ophthalmology. 2013;120:2611-9. https://doi.org/10.1016/j.ophtha.2013. 07.042 .

11. Shiode Y, Morizane Y, Matoba R, et al. The role of inverted internal limiting membrane flap in macular hole closure. Invest Ophthalmol Vis Sci. 2017;58:4847-55. https://doi. org/10.1167/iovs.17-21756.

12. Vogt D, Haritoglou C, Mautone L, et al. Premacular cells as source of neurotrophic factors in idiopathic macular holes. Curr Eye Res. 2020;45:1395-402. https:// doi.org/10.1080/ 02713683.2020 .1752389$.

13. Leisser C, Hirnschall N, Döller B, et al. Internal limiting membrane flap transposition for surgical repair of macular holes in primary surgery and in persistent macular holes. Eur JOphthalmol. 2018;28:225-8. https://doi.org/10.5301/ ejo.5001037.

14. Leisser C, Palkovits S, Hirnschall N, et al. One-year results after internal limiting membrane flap transposition for surgical repair of macular holes with respect to microperimetry. Ophthalmic Res. 2019;61:83-7. https://doi. org/10.1159/000489458.

15. Kang SW, Ahn K, Ham DI. Types of macular hole closure and their clinical implications. $\mathrm{Br} \mathrm{J}$ Ophthalmol. 2003;87:1015-9. https://doi.org/10.1136/bjo.87.8.1015.

16. SteelDH, DonachiePHJ, Aylward GW, BEAVRSMacular hole outcome group, et al. Factors affecting anatomical and visual outcome after macular hole surgery: findings from a large prospective UK cohort. Eye (Lond). 2021;35:316-25. https://doi.org/10.1038/s41433-020-0844-X.

17. Gekka T, Watanabe A, Ohkuma Y, et al. Pedicle internal limiting membrane transposition flap technique for refractory macular hole. Ophthalmic Surg Lasers Imaging Retina. 2015;46:1045-6. https://doi.org/10.3928/ 23258160-20151027-10.

18. Morizane Y, Shiraga F, Kimura S, et al. Autologous transplantation of the internal limiting membrane for refractory macular holes. Am J Ophthalmol. 2014;157:861-869.el. https://doi.org/10.1016/j.ajo.2013.12.028.

19. Casini G, Mura M, Figus M, et al. Inverted internal limiting membraneflap techniquefor MacUlar holesurgery without extra manipulation of the flap. Retina. 2017;37:2138-44. https:// doi.org/10.1097/IAE.0000000000001470.

20. Rossi T, Gelso A, Costagliola C, et al. Macular hole closure patterns associated with different internal limiting 
membraneflap techniques. Graefes Arch Clin Exp Ophthalmol. 2017;255:1073-8. https://doi.org/10.1007/s00417017-3598-9.

21. Essex RW, Kingston ZS, Moreno-Betancur M, Australian and New Zealand Society of Retinal Specialists Macular Hole Study Group, et al. The effect of postoperative facedown positioning and of long- versus short-acting gas in macular hole surgery: results of a registry-based study. Ophthalmology. 2016;123:1129-36. https://doi.org/10. 1016/j.ophtha.2015.12.039.

22. Baumann C, Dervenis N, Kirchmair K, et al. Functional and morphological outcomes of the inverted internal limiting
MEMBRANE flap technique IN small-sized and mediumsized MACULAR holes $\langle 400 \mu \mathrm{m}$. Retina. 2021;41:2073-8. https://doi.org/10.1097/IAE.0000000000003160.

23. Chou HD, Liu L, Wang CT, et al. Single-layer inverted internal limiting membrane flap versus conventional peel for small- or medium-sized full-thickness macular holes. Am J Ophthalmol. 2021;235:111-9. https://doi.org/10. 1016/j.ajo.2021.08.016.

Publisher's Note Springer Nature remains neutral with regard to jurisdictional claims in published maps and institutional affiliations. 